\title{
Development of a coconut- and palm-based fat blend for a cookie filler.
}

\begin{abstract}
Thirteen fat blends intended for cookie filler (CF) production that consist of 20-70\% palm mid-fraction (PMF), 20-70 \% virgin coconut oil (VCO), and 0-10\% palm stearin (POs) were developed based on the solid fat contents (SFC) of the fat portions extracted from five commercial CF samples: A, B, C, D, and E. A mixture design was applied for fat blend optimization, and the combination that best approached the target SFC values was composed of $70 \%$ PMF, $20 \%$ VCO, and $10 \%$ POs. The optimized coconut- and palm-based fat blend $(\mathrm{O}-\mathrm{CP})$ exhibited a steeper SFC profile, with $8.2 \%( \pm 0.2) \mathrm{SFC}$ at $25{ }^{\circ} \mathrm{C}$ (room temperature) and $0.2 \%( \pm 0.2) \mathrm{SFC}$ at $37{ }^{\circ} \mathrm{C}$ (body temperature); lower slip melting point of $34.0{ }^{\circ} \mathrm{C}( \pm 0.0)$; and a lower iodine value (IV) of $40.25 \mathrm{~g} / 100 \mathrm{~g}( \pm 1.04)$. In addition, O-CP contained higher proportions of medium-chain fatty acids (MCFA) and lauric acid (C12:0) of $3.2 \%( \pm 0.18)$ and $9.7 \%( \pm 0.43)$, respectively. In terms of its thermal profile, $\mathrm{O}-\mathrm{CP}$ showed no significant difference in terms of its crystallization range, $49.7^{\circ} \mathrm{C}( \pm 2.66)$ with the exception of sample $\mathrm{C}$, but it exhibited a smaller melting range, $65.8{ }^{\circ} \mathrm{C}( \pm 1.47)$, compared to the fat portions of the commercial samples. The ranges represented the span between the onset and offset temperatures of both crystallization and melting profiles as determined by differential scanning calorimetry.
\end{abstract}

Keyword: Cookie filler; Palm mid-fraction; Virgin coconut oil; Palm stearin; Mixture design. 